Original paper

\title{
Lymphovascular invasion on explant is associated with presenting tumor characteristics and not direct acting antiviral utilization in hepatitis $\mathbf{C}$ candidates undergoing liver transplantation
}

\author{
Paul Muna-Aguon', Meera Ramanathann', Myunghan Choi ${ }^{2}$, Mark Pedersen ${ }^{3}$, Anil Seetharam ${ }^{4,5}$ \\ ${ }^{1}$ Internal Medicine, Banner University Medical Center, Phoenix, AZ, United States \\ ${ }^{2}$ Arizona State School of Nursing and Innovation, Tempe, AZ, United States \\ ${ }^{3}$ Division of Digestive and Liver Diseases, University of Texas Southwestern, Dallas, TX, United States \\ ${ }^{4}$ Banner Transplant and Advanced Liver Disease Center, Phoenix, AZ, United States \\ ${ }^{5}$ University of Arizona College of Medicine-Phoenix, Phoenix, AZ, United States
}

\begin{abstract}
Aim of the study: Utilization of direct acting antiviral (DAA) therapy in candidates with well-compensated hepatitis $\mathrm{C}$ virus (HCV) cirrhosis and hepatocellular carcinoma (HCC) accruing end stage liver disease (MELD) exception points is highly variable among transplant centers based on center location, local organ procurement dynamics, $\mathrm{HCV}(+)$ organ availability, and patient preference. The association between DAA utilization prior to transplant and incidence of lymphovascular invasion on explant is unknown.

Material and methods: Retrospective evaluation from 2013-2017 of patients on a liver transplant (LT) waitlist with HCV-related cirrhosis, MELD-Na < 15, and HCC (within T2/Milan criteria). The cohort was divided into the pre-LT DAA treated group and untreated group with clinical/viral demographics collected. Tumor presenting characteristics, locoregional treatments, wait time to LT, dropout rates and explant pathology were compared.

Results: DAAs were used in 44 patients prior to LT (SVR12 of 37/44 [84\%]) and 19 left untreated with LT performed in $81 \%$ (51/63) of the waitlisted cohort. No significant differences were found between groups with regards to clinical/viral demographics, local-regional therapy (LRT) sessions, or frequency of lymphovascular invasion on explant. The untreated cohort had a higher rate of dropout (6.3\% vs. 3.2\%) $(p=0.041)$. On subgroup analysis of 51 subjects undergoing LT, AFP $>250 \mathrm{ng} / \mathrm{ml}(p=0.003)$ and multifocal HCC (> 1 lesion) $(p=0.006)$ were associated with lymphovascular invasion on explant while DAA therapy was not $(p=0.578)$.

Conclusions: DAA therapy for waitlist active HCV candidates accruing MELD exception points has no deleterious effects on bridging LRT, nor is it associated with increased frequency of lymphovascular invasion on explant. The latter appears driven by tumor related characteristics (AFP and number of lesions) irrespective of DAA utilization prior to LT. Key words: liver transplantation, hepatocellular carcinoma, lymphovascular invasion, hepatitis C virus infection.
\end{abstract}

Address for correspondence

Dr. Paul Alfred Muña Aguon, Internal Medicine, Banner University Medical Center, 1111 E. McDowell Road LL2, Phoenix, AZ 85006, United States, e-mail: paulaguon@gmail.com

\section{Introduction}

Introduction of highly effective and tolerable direct-acting antivirals (DAA) has improved waitlist mortality and lowered risk of disease progression among chronic hepatitis $\mathrm{C}$ virus (HCV) patients awaiting liver transplantation (LT) [1]. Optimal timing of DAA therapy in waitlist active candidates remains a subject of debate. Cost-effective analyses show an advantage for pre-transplant therapy $[2,3]$. In con- 
trast, a recent study identified higher quality adjusted life-years (QALYs) with DAA therapy after transplant, particularly when deferral maintains access to the expanded pool of $\mathrm{HCV}(+)$ donors for otherwise wellcompensated HCV-infected cirrhotics with hepatocellular carcinoma (HCC) [4].

Recent literature suggests an association between DAA therapy and the development of de novo and recurrent HCC [5-9]. The mechanism for increased risk is hypothesized to be due to alteration in natural killer (NK) cell function and dysregulation of immune surveillance with rapid viral clearance [10]. Initial reports of a link have not been corroborated by more recent investigation [11]. One study reported a lower incidence of HCC with DAA therapy [12] and another identified increased HCC incidence as a function of patient characteristics and lower screening intensity rather than DAA therapy itself [13].

DAA utilization in subjects awaiting LT with low "biologic" model for end stage liver disease (MELD) scores with HCC accruing exception points is highly variable among transplant centers. A recent investigation examined the impact of DAA therapy on HCC recurrence after local-regional therapy (LRT) and waitlist dropout among liver LT candidates with HCC [14]. In LT candidates with HCV and HCC with an initial complete response to LRT, DAA use was not associated with increased risk of HCC recurrence but rather a reduced waitlist dropout due to tumor progression or death. While previous investigation focused on waitlist dropout, little is known about the association of DAA therapy prior to transplant and incidence of lymphovascular invasion on explanted tissue at the time of LT and subsequent outcomes. Histological examination, especially assessment of lymphovascular invasion on pathologic explanted tissue, is important to assess recurrence risk and prognosis after LT [15]. Investigation evaluating microinvasion in patients with surgical resection for HCC found presence associated with lower 5-year survival (24-36\% vs. $61-63 \%)$ and earlier median recurrence (12 vs. 42.2 months). Invasion of tumor cells into the vasculature is hypothesized to serve as a route for intrahepatic metastasis leading to recurrence [16]. Renzulli et al. later evaluated radiographic features of microvascular invasion in HCV-cirrhosis patients with HCC nodules that developed before and after DAA therapy. They found that HCC nodules that developed after DAA therapy had a higher rate of microvascular invasion imaging features $(70.7 \%$ vs. $33.3 \%)$, which was indicative of more aggressive tumor [17]. The aim of this investigation was to examine the incidence of lymphovascular invasion on explant pathology in untreated and DAA treated $\mathrm{HCV}$ cirrhotic cohorts with HCC awaiting LT.

\section{Material and methods}

After obtaining institutional board approval, we performed a retrospective cohort study (2013-2017) of the University of Arizona College of Medicine-Phoenix LT program identifying consecutive subjects with: HCV related cirrhosis with "biologic" MELD-Na $<15$ and HCC presenting within T2/Milan criteria listed for LT. Subjects were excluded from analysis if: biologic MELD-Na $>15$ at the time of transplant listing, concomitant etiology for liver disease apart from $\mathrm{HCV}$ (concomitant viral, autoimmune, or inherited metabolic diseases) was identified; and if "downstaging" LRT of HCC to T2/Milan criteria was performed prior to listing. Treatment of HCV in waitlist active candidates at our center is determined on a case-bycase basis factoring candidate co-morbidities, baseline liver function, and patient preference. Center heterogeneity in utilization of DAA in this population allowed for identification of 2 cohorts: 1) untreated and 2) DAA treated HCV cirrhotics with HCC listed for transplant. Baseline demographics were collected on untreated and treated cohorts at the time of transplant listing including age, gender, ethnicity, BMI, biologic MELD-Na, as well as HCV genotype and viral load. For the cohort receiving DAA treatment, HCV regimen and sustained virologic response rates (SVR12) were recorded. For the entire cohort, presenting tumor variables - size and number of lesions, alpha-fetoprotein (AFP) - and number of LRT sessions/modalities were tabulated. Response to LRT was defined and tabulated in accordance with the Modified Response Evaluation Criteria in Solid Tumor (mRECIST) for HCC guidelines as applied by a United Network Organ Service (UNOS) certified abdominal radiologist [18]. Outcomes recorded included waitlist dropout or death and wait time to LT when applicable. Explant pathology reports of subjects receiving LT were reviewed for presence of lymphovascular invasion. Significant differences in continuous variables between untreated and treated cohorts were identified by $t$-test; categorical with chi-square test; $p<0.05$ (SPSS v24.0, Armonk, NY). Subgroup analysis for the cohort undergoing LT was performed using chi-square analysis to identify significant associations with lymphovascular invasion on explant pathology, $p<0.05$.

\section{Results}

\section{Patient population}

Sixty-three patients met inclusion and exclusion criteria, 19 untreated and 44 treated with DAA en 
route to liver transplant. Of the 19 untreated patients, 10 refused DAA therapy and consented for $\mathrm{HCV}(+)$ organ to increase candidate organ competitiveness after informed consent discussion, 6 refused and deferred consideration until after LT, and 3 had unstable financial/insurance coverage and were denied DAA therapy en route to LT. No significant differences existed between untreated and treated cohorts with regards to clinical demographic (Table 1). Untreated cohorts were listed at average "biologic" MELDs of $10.89 \pm 2.97$ compared to the treated cohort at an average of 10.02 $\pm 2.51(p=0.237) .44$ patients treated included 31 with genotype 1 , six with genotype 2 , and seven with genotype 3.31 patients with genotype 1 were treated with sofosbuvir/ledipasvir for 12 weeks; 4 patients with genotype 2 were treated with sofosbuvir/ribavirin and 2 with sofosbuvir/velpatasvir for 12 weeks; and 3 patients with genotype 3 were treated with sofosbuvir/ velpatasvir for 12 weeks while 4 patients were treated with sofosbuvir/ribavirin for 24 weeks. In subjects receiving ribavirin, subjects were started at $1200 \mathrm{mg}$ in 2 divided doses if $>75 \mathrm{~kg}$ or $1000 \mathrm{mg}$ in divided doses if $<75 \mathrm{~kg}$, and dose reduction to $600 \mathrm{mg}$ daily was allowed by treater discretion [19]. With respect to tumor characteristics, the largest tumor size was similar between cohorts; however, the DAA treated cohort had a significantly higher number of candidates with multifocal disease (Table 1).

\section{Response to DAA therapy}

Overall SVR12 for the treated group was 37/44 (84\%). No significant differences existed between untreated and treated cohorts with respect to genotype (genotype 1: $63 \%$ vs. $70 \%$, genotype $2: 16 \%$ vs. $14 \%$, genotype $3: 16 \%$ vs. $16 \%$, other: $5 \%$ vs. $0 \% ; p=0.482$ ), pre-treatment viral load (2.1 million $\mathrm{IU} / \mathrm{ml}$ vs. 2.74 million IU $/ \mathrm{ml}, p=0.652$ ), or previous treatment history (naive: $58 \%$ vs. $36 \%$, relapser: $32 \%$ vs. $34 \%$, non-responder: $10 \%$ vs. $30 \% ; p=0.176$ ) (Table 2). All treated patients received sofosbuvir-containing regimens for a minimum of 12 weeks. No subjects discontinued therapy while on treatment.

\section{Locoregional therapy treatment and dropout rates}

No significant differences were noted between untreated and DAA treated cohorts with respect to locoregional therapy modality which included transarterial chemo or radioembolization (or combination) while waitlist active for transplant (Table 3). There were no significant differences in number of LRT sessions
Table 1. Baseline demographics: Summary of clinical and tumor demographics between untreated and treated subjects with $\mathrm{HCV}$ and $\mathrm{HCC}$

\begin{tabular}{lccc}
\hline Parameter & $\begin{array}{c}\text { Untreated } \\
\text { pre-transplant } \\
(\boldsymbol{n}=19)\end{array}$ & $\begin{array}{c}\text { Treated } \\
\text { pre-transplant } \\
(\boldsymbol{n}=44)\end{array}$ & $\boldsymbol{P}$ \\
\hline Age at diagnosis of HCC & $60.05 \pm 5.8$ & $58.89 \pm 6.25$ & 0.490 \\
\hline Gender & & & 0.532 \\
\hline Male & $16(84 \%)$ & $34(77 \%)$ & \\
\hline Female & $3(16 \%)$ & $10(23 \%)$ & \\
\hline Ethnicity & & & 0.967 \\
\hline White & $13(68 \%)$ & $29(65 \%)$ & \\
\hline Black & $1(5 \%)$ & $3(7 \%)$ & \\
\hline Hispanic & $5(27 \%)$ & $12(27 \%)$ & \\
\hline BMl & $27.31 \pm 4.13$ & $27.99 \pm 4.84$ & 0.594 \\
\hline Creatinine & $0.85 \pm 0.23$ & $0.94 \pm 0.29$ & 0.282 \\
\hline Bilirubin & $1.54 \pm 1.01$ & $1.16 \pm 0.67$ & 0.142 \\
\hline INR & $1.27 \pm 0.26$ & $1.20 \pm 0.16$ & 0.168 \\
\hline Sodium & $137.89 \pm 2.54$ & $137.95 \pm 3.21$ & 0.943 \\
\hline MELD at diagnosis & $10.89 \pm 2.979$ & $10.02 \pm 2.51$ & 0.237 \\
\hline Number of lesions & & & 0.006 \\
\hline 1 & $11(58 \%)$ & $36(82 \%)$ & \\
\hline 2 & $8(42 \%)$ & $4(9 \%)$ & \\
\hline AFP at diagnosis & $0(0 \%)$ & $4(9 \%)$ & \\
\hline & $3.16 \pm 1.02$ & $2.86 \pm 1.03$ & 0.294 \\
\hline
\end{tabular}

Table 2. HCV treatment specifics: 44 out of 63 waitlist active HCV subjects underwent sofosbuvir-based direct acting antiviral therapy with an SVR12 rate of $84 \%$

\begin{tabular}{lccc}
\hline Parameter & $\begin{array}{c}\text { Untreated } \\
\text { pre-transplant } \\
(\boldsymbol{n}=19)\end{array}$ & $\begin{array}{c}\text { Treated } \\
\text { pre-transplant } \\
(\boldsymbol{n}=44)\end{array}$ & $\boldsymbol{P}$ \\
\hline Genotype & & 0.482 \\
\hline 1 & $12(63 \%)$ & $31(70 \%)$ & \\
\hline 2 & $3(16 \%)$ & $6(14 \%)$ & \\
\hline 3 & $3(16 \%)$ & $7(16 \%)$ & \\
\hline Other & $1(5 \%)$ & $0(0 \%)$ & \\
\hline Pretreatment viral load & 2178803.05 & 2748875.82 & 0.652 \\
& \pm 2275685.06 & \pm 5260029.37 & \\
\hline Prior treatment & & & 0.176 \\
\hline Naïve & $11(58 \%)$ & $16(36 \%)$ & \\
\hline Relapser & $6(32 \%)$ & $15(34 \%)$ & \\
\hline Non-responder & $2(10 \%)$ & $13(30 \%)$ & \\
\hline SVR12 & Not & $37(84 \%)$ & NA \\
\hline & applicable (NA) & & \\
\hline
\end{tabular}


Table 3. HCC treatment and transplant outcomes: Waitlist dropout due to tumor progression and death was seen in 4 patients in the untreated group and 2 patients in the treated group. Of the remaining 6 of 8 non-transplanted patients in the treated cohort, 2 were delisted due to active substance abuse and 4 remained waitlist active for transplant within T2/Milan criteria

\begin{tabular}{|c|c|c|c|}
\hline Parameter & $\begin{array}{l}\text { Untreated } \\
\text { pre-transplant } \\
(n=19)\end{array}$ & $\begin{array}{l}\text { Treated } \\
\text { pre-transplant } \\
(n=44)\end{array}$ & $P$ \\
\hline LRT modalities used & & & 0.104 \\
\hline TACE & $12(63 \%)$ & $21(48 \%)$ & \\
\hline TARE & $6(32 \%)$ & $10(23 \%)$ & \\
\hline Combination & $1(5 \%)$ & $13(29 \%)$ & \\
\hline Number of LRT sessions & & & 0.056 \\
\hline 0 & $1(5 \%)$ & $1(2 \%)$ & \\
\hline 1 & $5(26 \%)$ & $20(45 \%)$ & \\
\hline 2 & $2(10 \%)$ & $14(32 \%)$ & \\
\hline 3 & $7(37 \%)$ & $7(13 \%)$ & \\
\hline 4 & $3(17 \%)$ & $1(2 \%)$ & \\
\hline 5 & $1(5 \%)$ & $1(2 \%)$ & \\
\hline Tumor dropout & & & 0.041 \\
\hline No & $15(23.8 \%)$ & $42(66.7 \%)$ & \\
\hline Yes & $4(6.3 \%)$ & $2(3.2 \%)$ & \\
\hline Transplanted & $15(79 \%)$ & $36(81 \%)$ & NS \\
\hline $\begin{array}{l}\text { Lymphovascular invasion } \\
\text { on explant }\end{array}$ & 2 & 4 & 0.164 \\
\hline
\end{tabular}

TACE - transarterial chemoembolization, TARE - transarterial radioembolization

utilized while waitlist active for transplant. Dropout (progression of HCC beyond transplant criteria) rates were noted to be higher in the untreated cohort $(6.3 \%)$ compared to the DAA treated cohort $(3.2 \%)(p=0.04)$.

\section{Transplant rates}

Fifty-one of $63(81 \%)$ of subjects underwent LT (36/44 [81\%] treated, $15 / 19$ [79\%] untreated) at an average of $403.38 \pm 197.55$ days ( $396.22 \pm 196.39$-treated vs. $420.53 \pm 206.18$-untreated, $p=0.693$ ). All 4 of the non-transplanted patients in the untreated cohort died from tumor progression. With regards to the 8 nontransplanted subjects in the treated cohort, 2 had dropped out and died, 2 were de-listed secondary to active substance abuse, and 4 remain waitlist active for transplant within T2/Milan criteria.

\section{Risk factors for lymphovascular invasion}

In the 51 subjects ( 15 from the untreated and 36 from the treated group) undergoing LT there were no differences in incidence of lymphovascular invasion on explant: $2 / 15(13.3 \%)$ in the untreated cohort and $4 / 36(11.1 \%)$ in the treated cohort $(p=0.164)$. On further subgroup analysis stratifying for lymphovascular invasion, pretreatment AFP level $>250 \mathrm{ng} / \mathrm{ml}$ $(p=0.003)$ and multifocal HCC ( $>1$ lesion) $(p=0.006)$ was associated with presence of lymphovascular invasion on explant while DAA exposure was not $(p=0.578)$.

\section{Discussion}

Highly effective and tolerable DAA therapy has altered the landscape of HCV therapy even in subjects with decompensated cirrhosis [20]. Patients with advanced fibrosis and associated portal hypertension can experience rapid improvement in liver function parameters [21]. Such dramatic responses have influenced treatment algorithms in waitlist active candidates for LT, with many centers deferring treatment to facilitate access to the pool of $\mathrm{HCV}(+)$ donor livers and avoid the phenomenon of placing patients in "MELD purgatory" (improvement in liver parameters with associated reduction in MELD score/priority awaiting transplant) [22].

A number of clinical issues arise in the waitlist active candidate for transplant with HCV viremia, fibrosis, and HCC. Well-compensated patients have lower biologic MELD scores and the position on the waitlist has been historically driven by accrual of points with MELD exception [23]. Recently, a number of conflicting reports have evaluated the association between HCV treatment and incidence of HCC development in subjects with advanced fibrosis $[5,6,12]$. Application of DAA therapy in candidates with well-compensated HCV cirrhosis and HCC accruing MELD exception points is highly variable among transplant centers and often based on center location (local organ procurement dynamics/HCV(+) organ availability) and patient preference.

In this investigation, we examined differences in locoregional therapy, dropout rates, and incidence of lymphovascular invasion on explant in a cohort of otherwise well-compensated subjects with HCV related cirrhosis and HCC exposed and not exposed to DAA therapy. Similar to a previous study [14] we saw a slightly higher rate of HCC dropout in untreated subjects compared to those treated due to increased rates of tumor progression or death. Importantly, we found no significant differences in incidence of lymphovascular invasion between untreated and treated subjects who ultimately underwent LT despite the treated cohort having higher frequency of multifocal disease at the time of transplant listing. On subgroup analysis, we found that presence of lymphovascular invasion on explant was significantly associated with higher AFP 
level at HCC diagnosis and multifocal presentation rather than DAA therapy.

Additionally, our study showed lower SVR12 rates (84\%) when compared with historical rates seen in HCV patients without HCC. This is consistent with prior data showing that $\mathrm{HCV}$ patients with active HCC had higher treatment failure with DAA therapy. In a large study by the Veterans Affairs, Beste et al. found that SVR12 was achieved in $91.1 \%$ in non-HCC patients but only $74.4 \%$ with the presence of HCC [24]. It has been hypothesized that active HCC may serve as a "protected reservoir" for HCV infection, thereby reducing DAA efficacy, though mechanisms of viral evasion in the context of neoplasia need to be explored further [25].

Our study has limitations as it is retrospective, from a single center with a relatively small population. With regards to baseline demographics, we did not assess for the presence of concomitant non-alcoholic steatohepatitis (NASH) or alcohol as etiologies in subjects with $\mathrm{HCV}$; these co-factors may influence HCC biology and response to LRT. Another limitation is that our AFP measurement was cross-sectional at the time of diagnosis of clinical HCC diagnosis. Fluctuations in AFP level, not only with subsequent LRT but also with DAA therapy, may have prognostic value, and this should be the subject of a future prospective study. In addition, the decision to treat with DAA was left at the discretion of the treating hepatologist and, as such, it is plausible that subjects with a longer expected wait time were treated whereas those with higher priority (higher biologic MELD/exception point accrual) were left untreated, thereby introducing a classification bias. Nevertheless, mean MELD at listing was similar between cohorts and the entire cohort was subject to the same organ acceptance practices and regional organ procurement organization $(\mathrm{OPO})$ dynamics. Our results suggest that DAA therapy for waitlist active $\mathrm{HCV}$ candidates accruing MELD exception points is efficacious with no deleterious effects on bridging LRT or increase in frequency of lymphovascular invasion on explant. Lymphovascular invasion appears driven by tumor related characteristics (AFP and number of lesions) irrespective of DAA utilization prior to LT.

\section{Disclosure}

Authors report no conflict of interest.

\section{References}

1. Young K, Liu B, Bhuket T, et al. Improved liver transplant waitlist mortality and lower risk of disease progression among chronic hepatitis $\mathrm{C}$ patients awaiting liver transplantation after the introduction of direct-acting antiviral therapies in the United States. J Viral Hepat 2018; 26: 350-361.

2. Ahmed A, Gonzalez SA, Cholankeril G, et al. Treatment of patients waitlisted for liver transplant with all-oral direct acting antivirals is a cost-effective treatment strategy in the United States. Hepatology 2017; 66: 46-55.

3. Tapper EB, Catana AM, Sethi N, et al. Direct costs of care for hepatocellular carcinoma in patients with hepatitis $\mathrm{C}$ cirrhosis. Cancer 2016; 122: 852-858.

4. Salazar J, Saxena V, Kahn JG, et al. Cost-effectiveness of direct-acting antiviral treatment in hepatitis c-infected liver transplant candidates with compensated cirrhosis and hepatocellular carcinoma. Transplantation 2017; 101: 1001-1008.

5. Conti F, Buonfiglioli F, Scuteri A, et al. Early occurrence and recurrence of hepatocellular carcinoma in HCV-related cirrhosis treated with direct-acting antivirals. J Hepatol 2016; 65: 727-733.

6. Reig M, Mariño Z, Perelló C, et al. Unexpected high rate of early tumor recurrence in patients with HCV-related HCC undergoing interferon-free therapy. J Hepatol 2016; 65: 719-726.

7. Ravi S, Axley P, Jones D, et al. Unusually high rates of hepatocellular carcinoma after treatment with direct-acting antiviral therapy for hepatitis C related cirrhosis. Gastroenterology 2017; 152: 911-912.

8. Kozbial K, Moser S, Schwarzer R, et al. Unexpected high incidence of hepatocellular carcinoma in cirrhotic patients with sustained virologic response following interferon-free direct-acting antiviral treatment. J Hepatol 2016; 65: 856-858.

9. Tsai PC, Huang CF, Yu ML. Unexpected early tumor recurrence in patients with hepatitis $C$ virus-related hepatocellular carcinoma undergoing interferon-free therapy: Issue of the interval between HCC treatment and antiviral therapy. J Hepatol 2017; 66: 464.

10. Serti E, Chepa-Lotrea X, Kim YJ, et al. Successful interferon-free therapy of chronic hepatitis $\mathrm{C}$ virus infection normalizes natural killer cell function. Gastroenterology 2015; 149: 190-200.

11. ANRS collaborative study group on hepatocellular carcinoma (ANRS CO22 HEPATHER, CO12 CirVir and CO23 CUPILT cohorts). Lack of evidence of an effect of direct-acting antivirals on the recurrence of hepatocellular carcinoma: Data from three ANRS cohorts. J Hepatol 2016; 65: 734-740.

12. Calvaruso V, Cabibbo G, Cacciola I, et al. Incidence of hepatocellular carcinoma in patients with HCV-associated cirrhosis treated with direct-acting antiviral agents. Gastroenterology 2018; 155: 411-421.

13. Nahon P, Layese R, Bourcier V, et al. Incidence of hepatocellular carcinoma after direct antiviral therapy for $\mathrm{HCV}$ in patients with cirrhosis included in surveillance programs. Gastroenterology 2018; 155: 1436-1450.

14. Huang AC, Mehta N, Dodge JL, et al. Direct-acting antivirals do not increase the risk of hepatocellular carcinoma recurrence after local-regional therapy or liver transplant waitlist dropout. Hepatology 2018; 68: 449-461.

15. Piros L, Fehérvári I, Gorog D, et al. Examinations of factors influencing survival of liver transplantation for hepatocellular carcinoma: a single-center experience from Budapest. Transplant Proc 2015; 47: 2201-2206.

16. Lim KC, Chow PK, Allen JC, et al. Microvascular invasion is a better predictor of tumor recurrence and overall survival following surgical resection for hepatocellular carcinoma compared to the Milan criteria. Ann Surg 2011; 254: 108-113.

17. Renzulli M, Buonfiglioli F, Conti F, et al. Imaging features of microvascular invasion in hepatocellular carcinoma developed after direct-acting antiviral therapy in HCV-related cirrhosis. Eur Radiol 2018; 28: 506-513. 
18. European Association for the Study of the Liver and European Organisation for Research and Treatment of Cancer. J Hepatol 2012; 56: 908-943.

19. Hepatitis C Guidance 2018: AASLD-IDSA Recommendations for testing, managing, and treating adults infected with hepatitis C virus. Hepatology 2015; 62: 932-954.

20. Curry MP, O’Leary JG, Bzowej N, et al. Sofosbuvir and Velpatasvir for $\mathrm{HCV}$ in patients with decompensated cirrhosis. N Engl J Med 2015; 373: 2618-2628.

21. Deterding K, Höner Zu Siederdissen C, et al. Improvement of liver function parameters in advanced HCV-associated liver cirrhosis by IFN-free antiviral therapies. Aliment Pharmacol Ther 2015; 42: 889-901.

22. Gadiparthi C, Cholankeril G, Perumpail BJ, et al. Use of direct-acting antiviral agents in hepatitis $\mathrm{C}$ virus-infected liver transplant candidates. World J Gastroenterol 2018; 24: 315-322.

23. Ishaque $T$, Massie $A B$, Bowring $M G$, et al. Liver transplantation and waitlist mortality for HCC and non-HCC candidates following the 2015 HCC exception policy change. Am J Transplant 2019; 19: 564-572.

24. Beste LA, Green PK, Berry K, et al. Effectiveness of hepatitis $\mathrm{C}$ antiviral treatment in a USA cohort of veteran patients with hepatocellular carcinoma. J Hepatol 2017; 67: 32-39.

25. Singal AG, Lim JK, Kanwal F. AGA clinical practice update on interaction between oral direct-acting antivirals for chronic hepatitis $\mathrm{C}$ infection and hepatocellular carcinoma: expert review. Gastroenterology 2019; 156: 2149-2157. 